\title{
PERFORMANCE OF A NUCLEAR EMULSION SCANNER $\dagger$
}

\author{
PAUL V. C. HOUGH
}

The University of Michigan, Ann Arbor

Received 15 September 1959

A machine has been constructed which meets many of the needs for scanning of nuclear emulsions as emulsions are used in charged particle nuclear spectroscopy. Methods developed for track detection are reviewed briefly. In more

\section{Requirements for a Scanner to be used in Charged Particle Nuclear Spectroscopy}

In typical experiments in charged-particle nuclear spectroscopy a well collimated, monoenergetic beam of protons, deuterons, or alpha particles falls on a thin target and reaction particles at arbitrary angle relative to the incident beam are analyzed in momentum by a high-resolution magnet. Usually several hundred momentum channels are available at the image plane of the magnet and nuclear emulsions have often been used ${ }^{1,2}$ ) to record in ali these channels simultaneously.

The particular geometry at the image plane of the Michigan analyzer magnet is shown in

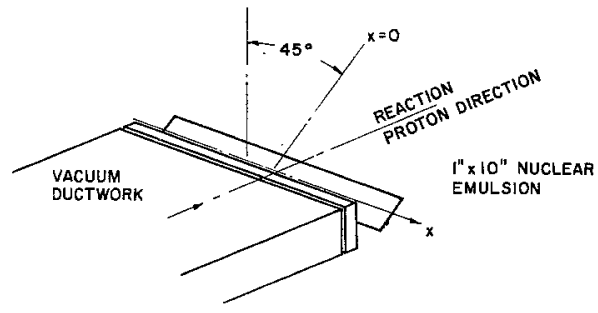

Fig. 1. The geometry at the image plane of the Michigan analyzer magnet.

fig. 1. The useful region of image plane extends from $x=+100$ to $x=-120 \mathrm{~mm}$ (in the coordinate system of the figure) and corresponds

$\dagger$ This work was supported by the Michigan Memorial Phoenix Project and by the U.S. Atomic Energy Commission. detail an account is given of the performance of the machine in scanning plates exposed at the Michigan $42^{\prime \prime}$ cyclotron over a seven months period. Finally, some special techniques which may have other applications are described.

to an energy variation equal to $15 \%$ of the central energy. The overall system resolution is about $20 \mathrm{keV}$ for $(\mathrm{d}, \mathrm{p}$ ) reactions and is limited primarily by a $15 \mathrm{keV}$ spread in energy of the $7.8 \mathrm{keV}$ deuteron beam on target. With this resolution, a $10 \mathrm{MeV}$ proton group will have a width $\Delta x \approx 4-5 \mathrm{~mm}$ at the emulsion.

With this background, we can give a reasonable set of requirements for an emulsion scanner used to read plates exposed at the magnet image plane:

\subsection{TRACK DETECTION EFFICIENCY}

A scanner need not have high absolute efficiency and probably any efficiency greater than about one-half would be quite satisfactory. However, the efficiency should be reproducible and constant for all emulsions used in a given experiment. Probably a fluctuation of $5 \%$ in efficiency would be tolerable for all nuclear structure experiments because of uncertainties in nuclear reaction theory.

\subsection{SPURIOUS COUNTING}

In exposures at the cyclotron for high energy proton groups (corresponding to low excitation of the residual nucleus) even long exposures will still yield regions on the emulsion where no

1) Buechner, Browne, Enge, Mazari and Buntschuh, Phys. Rev. 95 (1954) 609.

2) Bach, Childs, Hockney, Hough and Parkinson, Rev. Sci. Tnstr. 27 (1956) 516. 
tracks at all are found. This observation leads to the difficult conclusion that a scanner should introduce no spurious counts. More realistically, 10-20 tracks are certainly required to establish the presence of a proton group and therefore a spurious count of $3-4$ over a $4-5 \mathrm{~mm}$ width of emulsion is probably satisfactory.

\subsection{SCANNING SPEED}

It has proved possible at the Michigan cyclotron to expose five to ten $1^{\prime \prime} \times 10^{\prime \prime}$ nuclear emulsions per day for a number of days. Since an accelerator will normally be used for other experiments, this is probably an upper limit to the production rate for a single magnet image plane. On the other hand, a multigap magnet ${ }^{3}$ ) will increase this rate by an order of magnitude. It appears then that a scanner should be able to read 25-100 $1^{\prime \prime} \times 10^{\prime \prime}$ nuclear emulsions per day under the rather extreme demands placed on it by a multi-gap magnet installation.

\section{Track Detection Methods}

Details of electronic and optical methods developed to detect slow proton tracks are given elsewhere $\left.{ }^{4}\right)$. Here we only want to review the logic of the scheme so as to make clear the reasons for the performance characteristics listed in the next section.

3) W. W. Buechner, private communication.

4) Hough, Koenig and Williams, Electronics (McGrawHill, March 27, 1959).

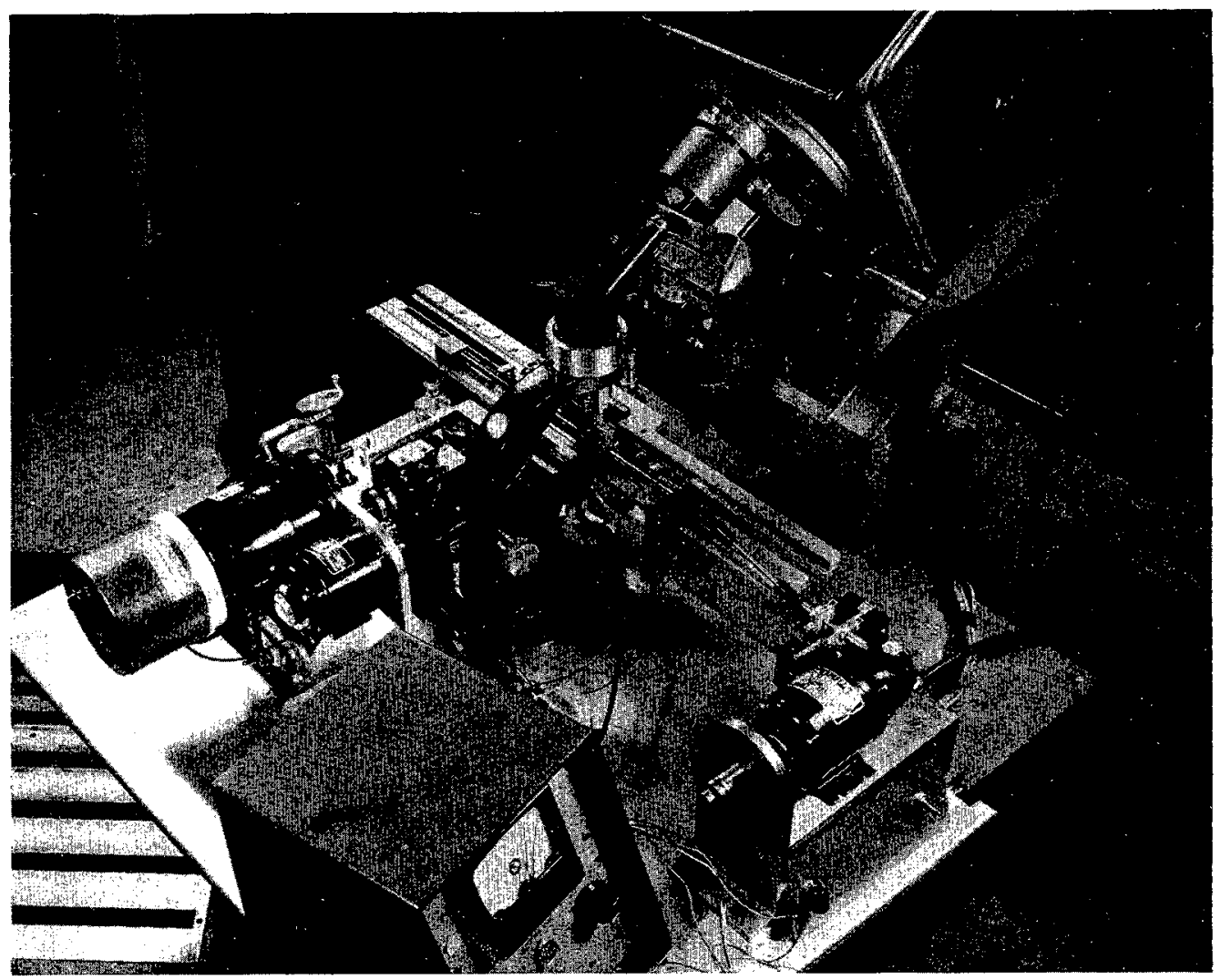

Fig. 2. The scanner microscope, showing the motor driven stage. 




Fig. 3. A typical emulsion scene photographed from a television receiver wired to the Image Orthicon Television Camera. For an explanation of the white marks beside the tracks and at the bottom of the field see section $2, \S \S 3$ and 4 .

\section{I. PATTERN OF SEARCH OVER THE EMUISION}

A motor driven stage (fig. 2) moves the $1^{\prime \prime} \times 10^{\prime \prime}$ emulsion under a microscope in a series of traverses of the short dimension, each traverse followed by an advance of the plate. During a traverse the stage moves continuously, but by means of a pulsed light source 140 separate fields of view are presented to a television camera and associated computer for analysis. Each field of view is $0.12 \mathrm{~mm} \times 0.18$ $\mathrm{mm}$; corresponding points in successive fields are separated by $0.15 \mathrm{~mm}$; and successive traverses are separated by $0.25 \mathrm{~mm}$. Normally the track count for four traverses is recorded as a single datum and so points are available for each $\mathrm{mm}$ along the emulsion. However, because of the gaps between fields and between traverses, each point corresponds to a search area only $0.56 \mathrm{~mm}$ wide (extending across the plate). The emulsion is examined at only one depth, midway between top and bottom of emulsion, so scanning is effective only for tracks which penetrate the emulsion completely.

\subsection{THE DETECTION OF EMULSION GRAINS, ESPECIALLY THE GRAINS MAKING UP TRACKS}

A standard Leitz 22x oil immersion objective and $10 \mathrm{x}$ eyepiece are used to project an image of the emulsion scene onto an Image Orthicon television camera tube. Fig. 3 shows a typical emulsion scene photographed from a television receiver wired to the camera. (The white dots to the right of the tracks are explained in paragraph 3 below, and the white splashes at the bottom in paragraph 4.) Two alterations have been made in the microscope optics: 
It is found that the contrast is considerably enhanced by blocking off a band of light entering the microscope condenser in a plane containing the tracks and the optic axis; this leaves two "crossfire" beams illuminating the emulsion.

Also it is found helpful to introduce a cylindrical lens of radius of curvature $15 \mathrm{~cm}$ adjacent to the eyepiece so that the image of each grain becomes a short line roughly parallel to most tracks. This trick tends to patch gaps in tracks without seriously patching together background grains into false tracks.

Kodak NTB2 or Ilford G-special emulsion are used to provide strong tracks with tolerable background grain density.

The portions of the scene detected by a special screening circuit are shown in fig. 4 . A narrowness criterion is incorporated in the screening circuit so that any large-area opacities which may occur are ignored.

\subsection{THE RECOGNITION OF A TRACK BY ITS CONTINUITY}

Delay and coincidence units are used to establish the existence of a grain just one scan line after a previous grain. A continuous track will of course give the required coincidence. The coincidence resolving time is chosen so that tracks at angles up to $上 45^{\circ}$ to the vertical will still give a coincidence. An actual counting operation is performed along a track by this (delay + coincidence) technique; in fact a continual testing of background grains for the possibility that they are linked to form tracks proceeds at all lateral positions in the field of view. An output pulse which we name "track segment pulse" is produced when the count along any track reaches 16 , and for each $\mathbf{8}$ counts thereafter. Since the number of television scan lines used per field is about 180 , the number of track segment pulses for a track crossing the entire field is 23. A more typical number of

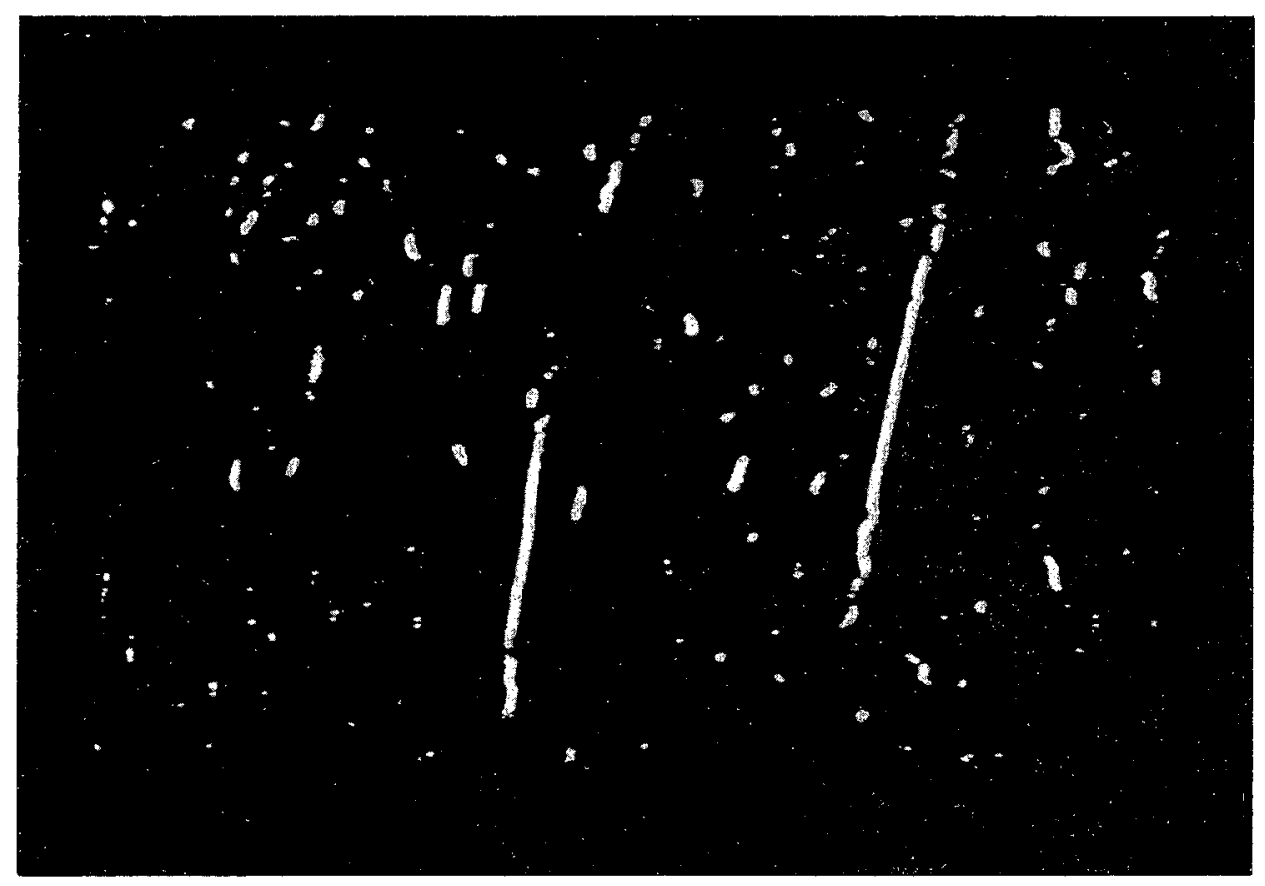

Fig. 4 . The portions of the scene of fig. 3 which are detected by a special screening circuit. 


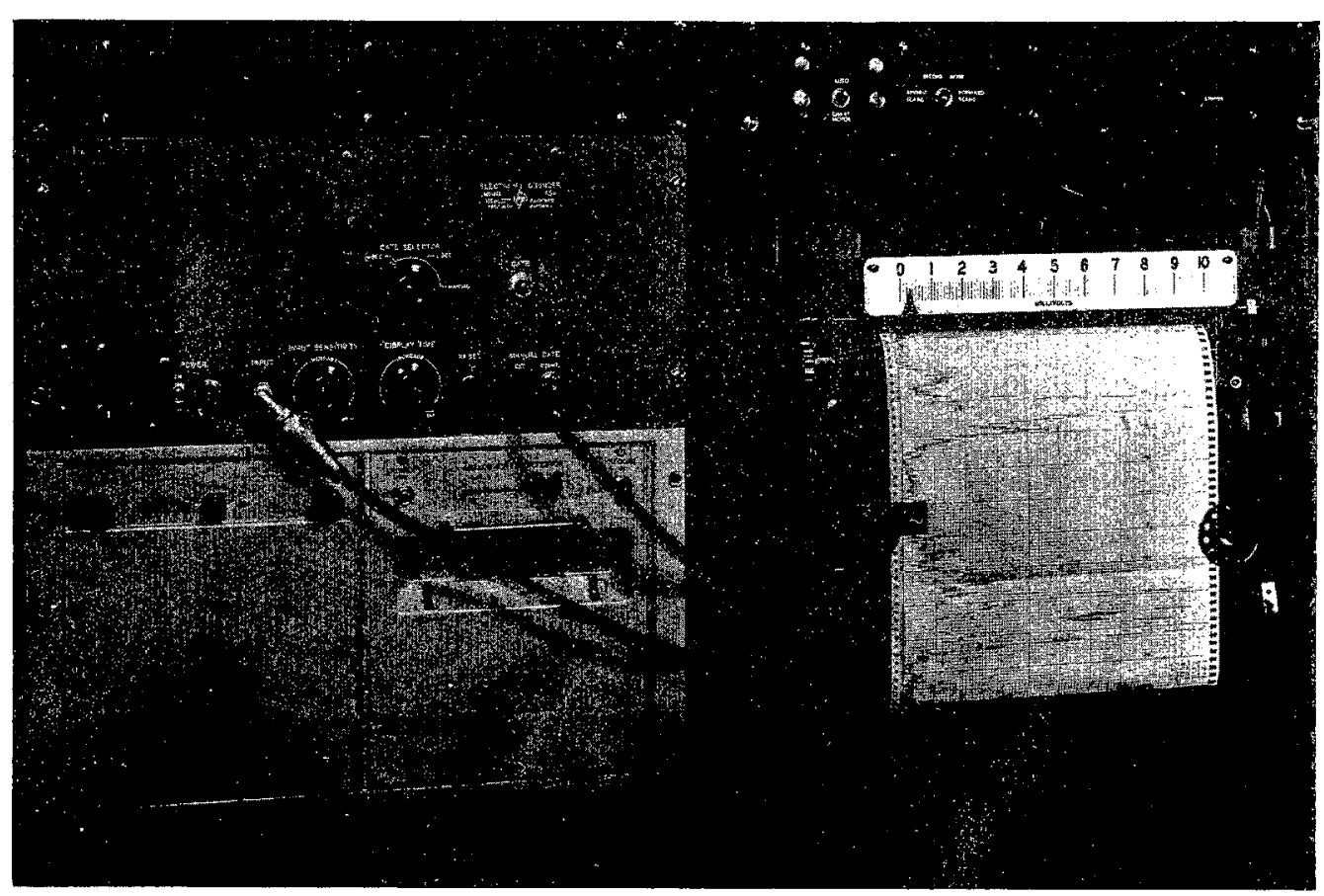

Fig. 5. Track counts accumulated for four traverses are printed on paper tape, and also plotted with appropriate error bars on a chart recorder. The strong groups are plotted with a factor 10 scale change.

track segment pulses for a track is $8-10$ as shown in a display of these pulses as as white dots to the right of each track in fig. 3. The doubling of pulses for the left track occurs because the exposure extends over several television scanning periods and the count along the track has begun at two different points in the different periods.

\subsection{GENERATION OF A SINGLE OUTPUT PULSE PER TRACK}

Owing to different quality of development for different tracks andi to more or less favorable location of a track in the field, the number of track segment pulses obtained per track varies widely. In order to obtain one count for each track the following technique is used: an oscilloscope beam is swept along a fixed line in synchronism with the horizontal deflection of the television scanning beam. Whenever a track segment pulse occurs the oscilloscope beam is intensified and a flash of light is produced. For vertical tracks all flashes for one track occur in one spot. The oscilloscope screen is projected back into the television camera at the bottom of the field of view. The camera tube integrates the light from the flashes originating in any one spot, i.e., from one track. Finally, a single television scan line through the spots is devoted to reading out the number of spots and therefore the number of tracks. (The two spots for the two tracks of fig. 3 appear dimly at the bottom of the figure. The brightness distribution is distorted badly by the television receiver; as actually used, the spots have a uniform intensity over a vertical distance equal to that of the black band of the figure. The readout line is centered in the black band.) It is possible to set a discriminator level on the final spot readout to select only tracks with more 
than a specified number of track segment pulses, usually 5 or 6 .

\subsection{FINAL OUTPUT}

The number of spots detected is accumulated in a scaler until four traverses across the plate width are completed. Then this number is printed on paper tape and plotted on a chart recorder as shown in fig. 5 . The error bars are obtained via a square-root potentiometer mounted on the shaft of the chart recorder. A recorder scale change from 200 tracks to 2000 tracks full scale can be seen for three intense groups in fig. 5 .

\section{Scanner Performance}

3.1. TRACK DETECTION: ABSOLUTE EFFICIENCY AND REPRODUCIBIIITY

On comparing the scanner with a human observer for a series of proton groups and a series of plates, the scanner count may be found anywhere between $80 \%$ and $120 \%$ of the human count, for equal search areast. The machine-human ratio changes only slowly with position on the plate so that peak positions and peak contours are reproduced fairly accurately, but the failure of the machine to maintain constant efficiency is a major defect.

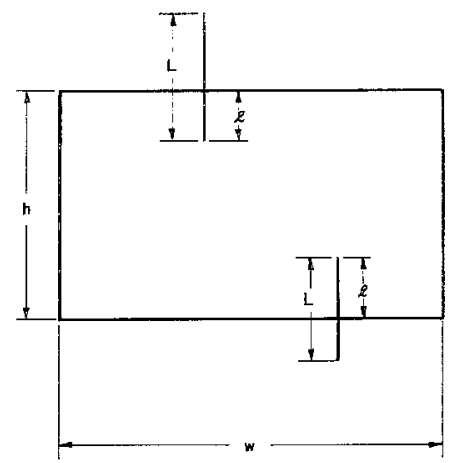

Fig. 6. Two tracks of length $L$ shown in extreme positions for detection. The minimum length within the field required for detection is $l$.

The difficulty has been traced to the use of isolated fields of view as the search pattern for the scanner. Referring to fig. 6 , define $w=$ width of field of view, $h=$ height of field of view, $L=$ length of track in good focus (as judged by the electronics), $l=$ length of track within the field of view required for the track to count. Evidently if the upper end of a track falls within the area $w \times[h+(L-l)-l]$ the track will count, and therefore the effective search area is increased over the geometrical area $w \times h$ by the factor

$$
\frac{h+(L-l)-l}{h} .
$$

The parameter $l$ is easily held constant, usually at the value $l=\frac{1}{4} \mathrm{~h}$, but $L$ is subject to fairly wide variation with quality of emulsion development and especially with slight changes in the optical and television systems. This variation in $L$ and the corresponding variation in search area is responsible for the major part of the observed variation in machine detection efficiency.

Simply considering the scanner as a counter, this problem of searching in isolated fields shows up clearly. Because tracks can have any fraction of their length inside the field of view the pulse height distribution from tracks observed in a collection of isolated fields necessarily extends down to zero. Now since the scanner uses a simple level discriminator to record all track pulses above a certain arbitrary height, if the mean pulse height changes (i.e., the mean track length as judged by the electronics changes), the fraction of pulses above a fixed level will in first order respond linearly. The scanner, regarded as a counter, is a counter without a plateau.

The scanner is still quite useful in searching for new particle groups and for identification of the mass of the target nucleus responsible for new groups by reading spectra at a number of reaction angles. However, human scanning is needed for absolute counts over the peaks.

Recently, an alternate scanner search pattern of continuous strips has been provided at a factor 4 reduction in speed. The results of two

$\dagger$ A smaller fluctuation of $\pm 5 \%$ is quoted in ref. ${ }^{4}$ ), but this is incorrect. 
such machine scans are compared with a hand scan in fig. 7 , showing a much more constant

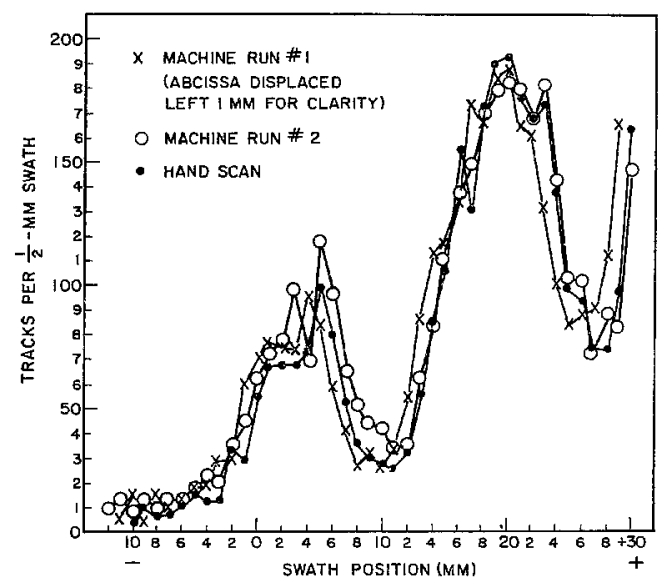

Fig. 7. Preliminary results for continuous strip scanning. Two machine scans are compared with a hand scan of the same region.

detection efficiency. It seems that it may be possible to carry out also by machine the absolute counting required.

\subsection{SPURIOUS COUNTS}

The background count introduced by the machine averages about one per plotted point, i.e., per geometrical search area equal to a strip $0.56 \mathrm{~mm}$ wide extending across the 1-inch dimension of the plate. Most of this machine background arises from neutron recoil tracks which are easily rejected by a human observer.

At first, emulsion surface marks often introduced whole blocks of spurious counts. Fortunately it was found that surface marks are easily removed by rubbing off the top few microns of processed emulsion with paper tissue soaked in methyl alcohol. This cleaning process is now followed routinely and is necessary for low background.

The scanner background is low enough so that it is not a limitation in nuclear spectroscopic applications.

\subsection{SHORT-TERM AND TIME-AVERAGED SCANNING RATES}

We have noted above that a "point" plotted for each $\mathrm{mm}$ of the analyzer magnet image actually corresponds to the geometrical search area of a strip $0.56 \mathrm{~mm}$ wide. So about half the plate area is normally scanned.

The scanner plots four points per minute and therefore reads the $220 \mathrm{~mm}$ useful range of an image plane in 55 minutes. Normal cleaning, recording and checking operations between plates occupy another 20 minutes, so that in steady, trouble-free scanning plates are read at the rate of one every hour and 15 minutes.

Averaged over an eight hour day it is possible to read five image planes $\left(1^{\prime \prime} \times 10^{\prime \prime}\right.$ plates) and fairly easy to read four. For the past few months it has proved useful to employ undergraduate assistants to put on a second shift from 5 to 11 P.M. in. which case another three or four plates are normally read. In sum, 7-9 image planes can be read per day, or about 1800 plotted points. By comparison a human scanner will produce between 100 and 150 plotted points per day.

Because of operator fatigue, the machine rate could probably not be maintained for months. It has not been necessary to try, because on the one hand large scale cyclotron exposures are ordinarily interspersed with exploratory runs or experiments with counters, and on the other, it has unfortunately been easy for the machine to saturate the three available human scanners with absolute counting jobs.

\subsection{PROBLEMS OF OPERATION AND MAINTAINANCE}

The whole system uses 635 tubes: 128 in locally designed and built circuits, 154 in the television system, 132 in broad band amplifiers, 103 in various other commercial units, and 118 in power supplies. In view of this complexity, arrangement is made to check the performance of any part of the scanner by switching in a synthetic signal which imitates a straight, vertical, continuous track. As a final integral check, at the beginning and end of each scanning 
period a standard proton group is counted and the machine required to produce a count within $\pm 10 \%$ of the correct value.

During scanning runs, the track segment pulses defined in section 2, paragraph 3 , are displayed continuously on a television screen. The resulting pattern changes at a $60 \mathrm{cps}$ rate, but it is still possible to detect tracks by eye and for low track densities to verify the performance of the machine track by track. At higher track densities visual checking becomes impossible, but a gross failure of any part of the machine is usually obvious.

The failure rate of the circuitry built in our laboratory is about the same as the failure rate of the television system, and for about the same number of tubes. Tube failure has been the most frequent but not the most annoying cause of circuit failure. More troublesome have been the development of bad contacts at solder joints and the development of high frequency oscillations in portions of the circuit which had been free of difficulty since construction, often a year or two earlier. The effort and especially the quantity of highly skilled effort which can go into uncovering the cause of failures has encouraged the development of very complete records of normal circuit performance, especially

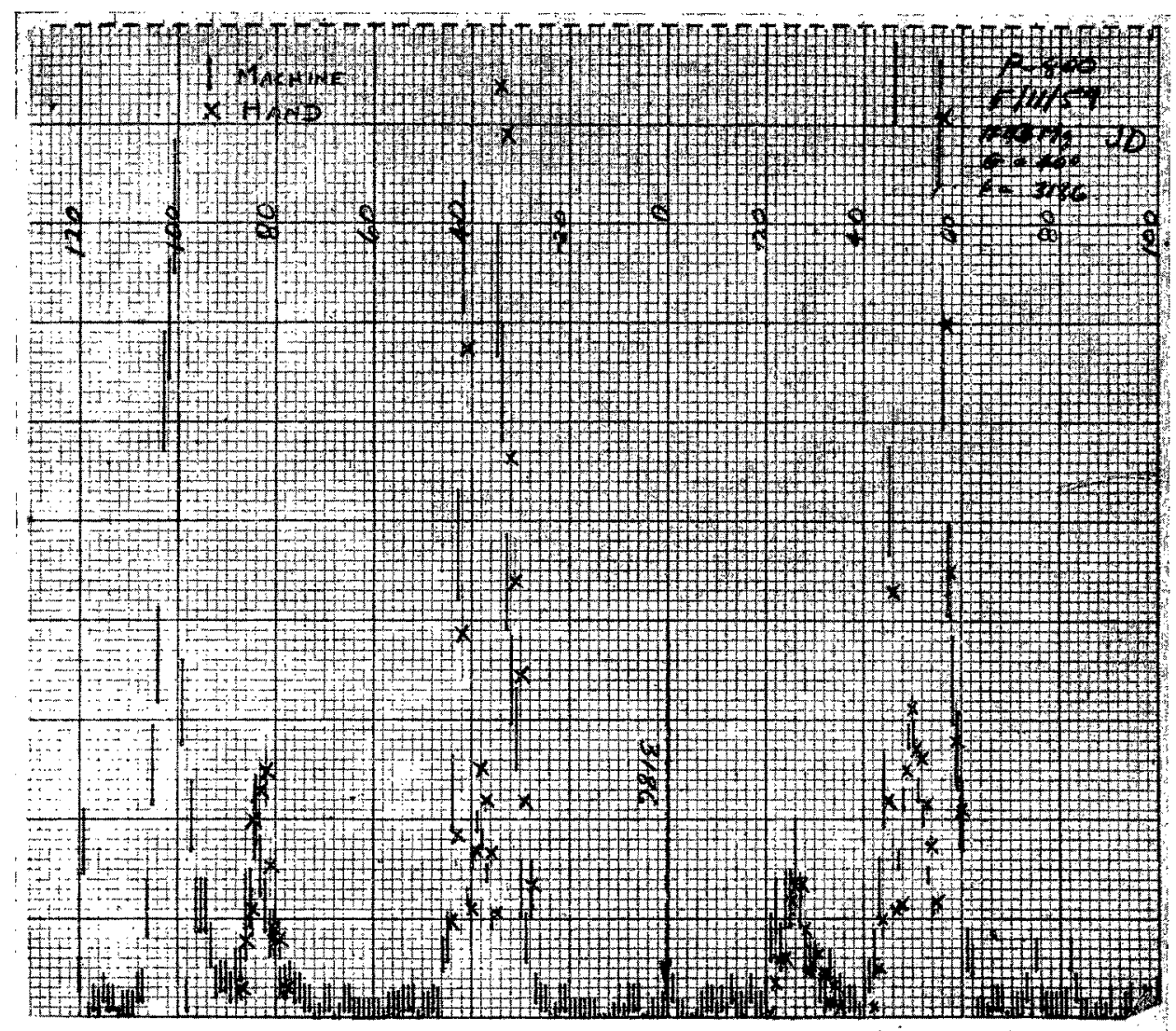

Fig. 8. A typical machine scan. Iand counts over the peak are also shown. The lateral shift of hand and machine data is due to a different convention in reading the left end of the plate. 


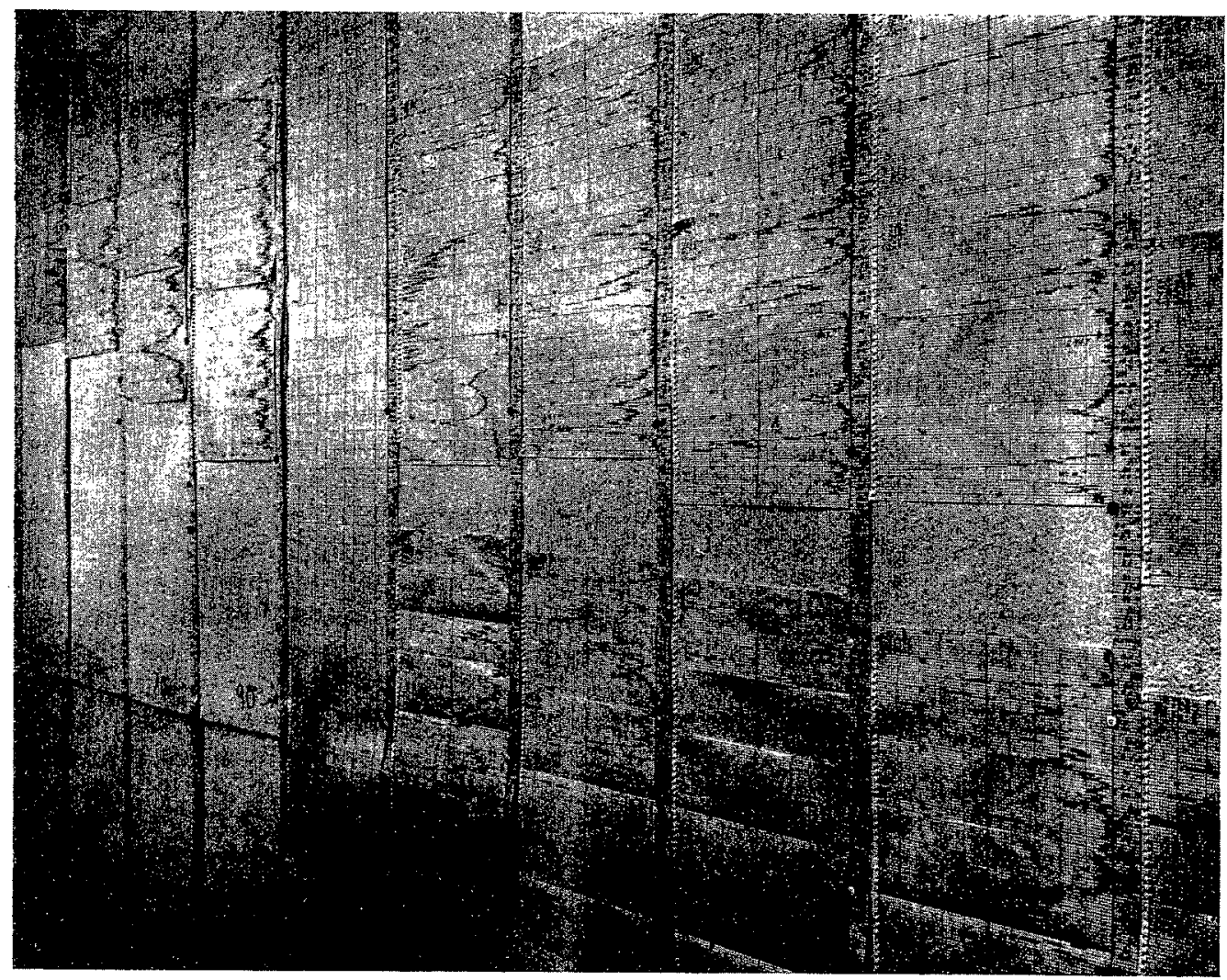

Fig. 9. An analysis board used to keep track of an investigation of the level structure of $\mathrm{Mg}^{25}$. For details see the text.

a comprehensive collection of oscilloscope drawings.

\subsection{RESULTS OBTAINED OVER A SEVEN MONTHS PERIOD}

Over the first seven months of 1959 the scanner read $2061^{\prime \prime} \times 10^{\prime \prime}$ plates exposed at the cyclotron. About 30 piates were not read, 4 because of a development failure and the rest because similar exposures had been read by machine and the existence and rough intensities of the various proton troups had been established. At the beginning of the period scanning requests were met with great difficulty and often several days would elapse while operational difficulties were traced down. A shift from
Kodak NTB to Kodak NTB2 (or Iiford Gspecial) emulsion, the development of a really reliable focus control for the microscope, and a number of small engineering improvements have made scanning much easier so that by the end of the period relatively untrained personnel could read plates on demand within the performance specifications we have quoted.

Fig. 8 shows a chart of a machine scan, with hand counts over several peaks also plotted. The lateral shift of hand and machine data is due to a different convention in reading the left end of the plate.

Finally, as a general indication of the kind of work done by the scanner, we show in fig. 9 a photograph of part of an analysis board used to 
keep track of an investigation of the level structure of $\mathrm{Mg}^{25}$. The board is divided into halves, the left half devoted to proton groups of energy between 4 and $8 \mathrm{MeV}$, the right half to groups between 8 and $14 \mathrm{MeV}$. In each half, channels are established for mounting spectra of reaction protons observed at angles $10^{\circ}, 20^{\circ}$, $30^{\circ}, 40^{\circ}, 50^{\circ}, 70^{\circ}$ and $90^{\circ}$. (About twice as many angles are run for an angular distribution.) In each channel the same energy scale is established: the scale is non-uniform, with a rate of change of energy with position at any energy approximately equal to that of a scanner chart run at that energy. Therefore overlapping charts fit together to give continuous spectra. The analysis board is mainly a bookkeeping aid, useful for planning exposures and deciding what work is completed. By looking at the forwardangle charts the qualitative stripping angular distribution of a particular group is clear, and by inspection of the large-angle charts, the change in proton energy with angle is evident and therefore even by inspection one can make a close estimate of the mass of the target nucleus responsible for the group. The energies of the usual contaminant groups from carbon, oxygen and hydrogen can be plotted on the board once for all.

\section{Some Special Techniques}

\subsection{AUTOMATIC FOCUS CONTROL FOR A MICROSCOPE}

In scanner operation it is necessary to control the position of the microscope objective relative to the top surface of the emulsion to within \pm 10 microns. This particular number follows from the need to keep a focal region about 20 microns deep entirely within a thickness of processed emulsion equai to $\mathbf{4 0}$ microns. Without focus control, reasonable care in leveling the microscope stage will reduce variations in objective-emulsion separation to about 50 microns. This residual variation is of two types: (1) the focus changes abruptly by 15-20 microns according to the direction of traverse; (2) the focus changes slowiy with lateral position due to a nonzero angle between the plane of the top surface of the emulsion and the plane of the stage motion.

A very simple mechanical control reduces focus variations to about \pm 5 microns. In fig. 10 , spring $\mathrm{A}$ is used to load the lower part of the objective against the emulsion. The tungsten carbide ball $\mathrm{B}$ is rigidly attached to the loaded objective by the heavy steel piece $\mathrm{C}$ and contitutes the bearing point against the emulsion.

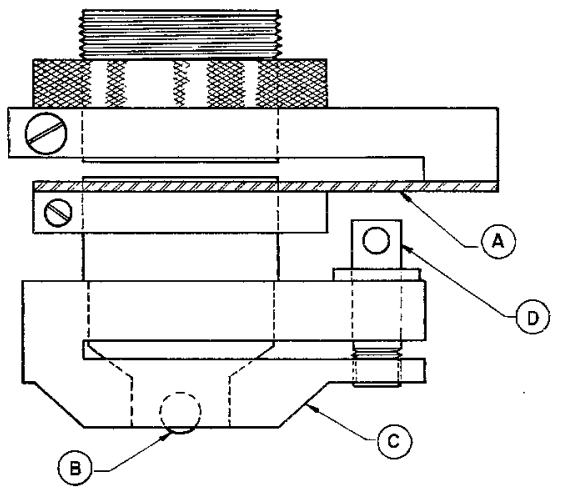

Fig. 10. A simple mechanical focus control which maintains the plane of best focus a fixed distance below the emulsion surface with an accuracy of about \pm 5 microns.

The bearing point is displaced from the optic axis of the objective by about $4 \mathrm{~mm}$, perpendicular to the plane of the paper. The screw $D$ is turned with a small removable wrench to distort piece $\mathrm{C}$ by a mil or two, moving the ball B up or down relative to the objective and thereby adjusting the depth at which the focus is controlled. In making the initial adjustment of the control the objective is lifted via the tungsten carbide bearing about 6 mils from its equilibrium position by raising the microscope stage. Then a restraining cam (not shown) is installed to catch the objective after a drop of 2 mils. This allows the objective to slide smoothly onto the emulsion again after leaving it at the end of scanning traverses.

\subsection{ELECTRONIC CONSTRAST ENHANCEMENT FOR FASTER HUMAN SCANNING}

Given a television system to look at emulsions, the various types of electronic analysis 

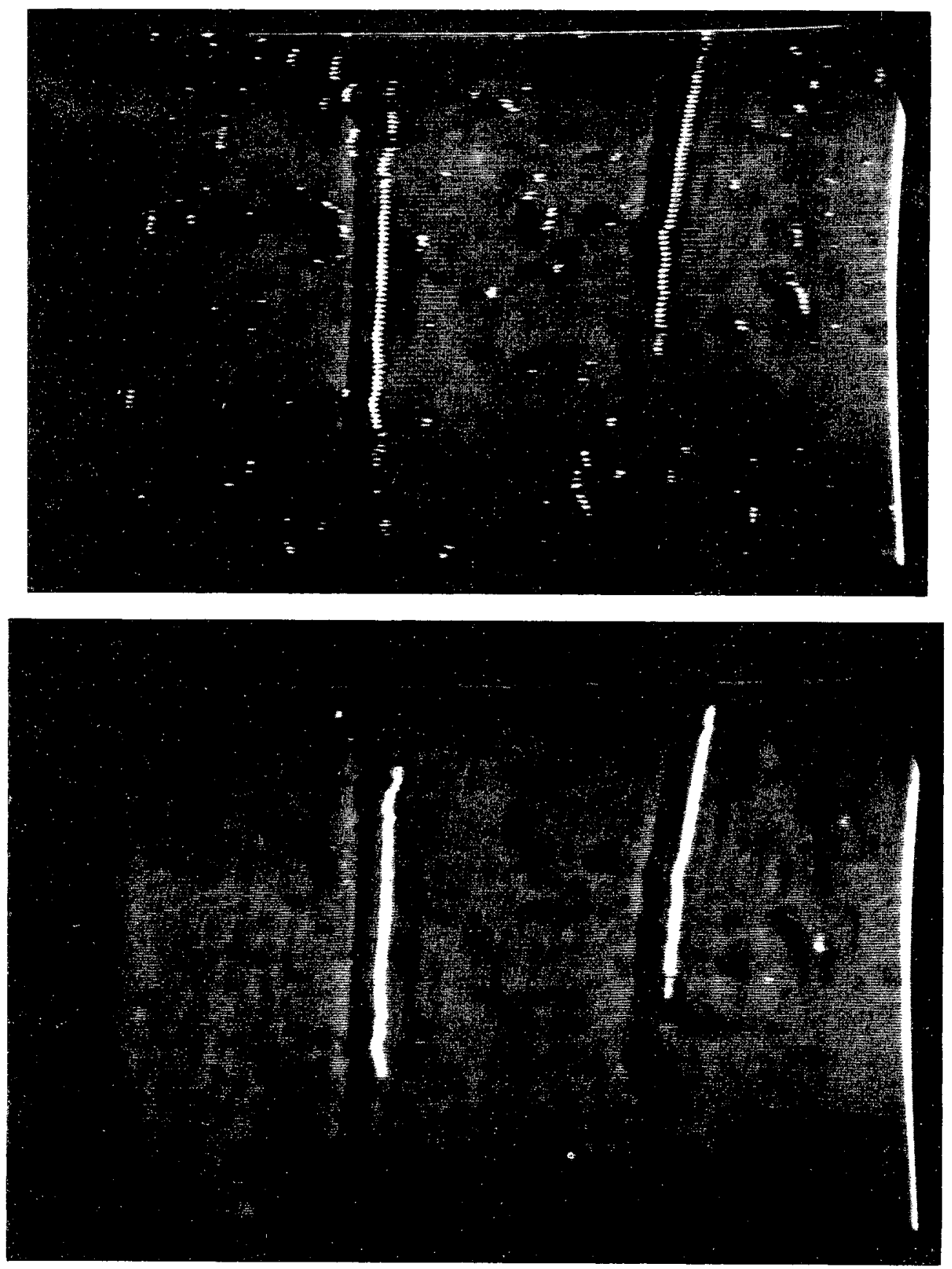

Fig. 1la and b. A normal scene with two possible forms of electronic contrast enhancement. 
used in wholly automatic scanning can be presented aiso on a television receiver for easier human scanning. Without going into detail, fig. 11 shows a normal scene with two possible forms of electronic enhancement. A defect in some applications would be the discrimination likely to occur against tracks which are parallel to the television scan lines.

\section{Acknowledgements}

It is a pleasure to thank G. R. Garrison, Director, and F. M. Remley, Technical Director, of the University of Michigan Television station for generous assistance. The excellent technical development work of J.A. Koenig was essential at every stage of the investigation. D. E. Damouth has been responsible for the development of continuous-strip scanning which shows such promise for reproducible efficiency. The contribution of R. O. Winder, W. Williams, and $B$. Cosby in the creation of certain component parts of the scanner is gratefully acknowledged. 